\title{
Symbiosis
}

\section{Vascular effects of cannabis: Case report and review of literature}

\author{
Homa Timlin ${ }^{* 1}$, Kirthi Machireddy ${ }^{2}$, Luc Nguyen ${ }^{3}$, Hilda T Draeger ${ }^{4}$ \\ ${ }^{1}$ Division of Rheumatology, Johns Hopkins University \\ 2University of the Sciences, Philadelphia, PA, USA \\ ${ }^{3}$ Division of Rheumatology, University Medicine Associates \\ ${ }^{4}$ Division of Rheumatology, UT Health San Antonio
}

Received: March 9, 2017; Accepted: April 10, 2017; Published: May 4, 2017

*Corresponding author: Homa Timlin MD MSc, Johns Hopkins Division of Rheumatology, 5501 Hopkins Bayview, Circle/Asthma and Allergy Building, Suite 1B.1, Baltimore, MD 21224.

\begin{abstract}
Marijuana is the 3rd most popular recreational drug and the use of recreational and medical marijuana has been legalized in several states. Knowledge of both acute and chronic adverse effects of cannabis is essential when counseling the public. We report 2 cases who developed arteritis and Raynaud's as a squeal of long term cannabis use and provide a literature review.
\end{abstract}

\section{Introduction}

Marijuana was first suggested to be used for medicinal benefit almost 4000 years ago by Chinese emperors [1] and from 1851 to 1942; marijuana was listed as a medication [2]. Despite, this history, marijuana was regarded as a dangerous substance with no additional medical benefit by the United Nations Single Convention on Narcotic Drugs of 1961 [3]. Several countries have legalized use of medical marijuana. Cannabis use has doubled over the past decade [4]. In 2014, an estimated 22 million people were using the drug [5]. With increasing legislation for the recreational and medicinal use of cannabis, knowledge of the short and long term adverse effects of cannabis is paramount for appropriate counseling. We report 2 cases of cannabis induced arteritis and Raynaud's that occurred after long term use of cannabis.

Key words: Cannabis, Vasculitis, Raynauds, Thromboangiitis obliterans, Connective tissue disease.

\section{Case One}

A man in his 50 's, was admitted with a six week history of cool, painful, purplish fingertips of both hands. He was diagnosed with presumed new onset Reynaud's one month previously which did not respond to nifedipine. Subsequently he was hospitalized with progressive symptoms, and tissue necrosis affecting the tips of digits $2-5$ of both hands as well as new purplish discoloration of two of his toes. Preceding symptoms included decreased appetite and a several pound weight loss. He had forty pack year history of tobacco and had quit 8 years previously At the time of admission, he had been smoking marijuana on a daily basis for several years, up to ten joints a day, and reported a recent increase in use due to hand pain. He denied other illicit drugs. Nifedipine was his only medication.
Physical examination revealed a middle aged man who appeared well. His vital signs were stable. The tips of fingers 2-5 bilaterally were cool and tender to palpation. Eschar formation was present on all digits distal to the DIP joints sparing the right fourth finger and bilateral thumbs with purplish discoloration extending proximal to the eschar. Radial pulses were strong, but ulnar pulses were absent bilaterally with positive Allen's test. The patient also had tenderness and purplish discoloration of his right fourth and fifth toes. No neurologic deficits or synovitis was appreciated.

A complete blood cell count was only notable for platelets of 456,000 . His serum creatinine and urinalysis were normal. Liver studies were significant for a mildly elevated ALT of 39 and his ESR was 31. Urine drug screen was positive for cannabinoid. Serum complements were slightly elevated with a C3 of 175 and C4 of 56. Studies for hepatitis $\mathrm{B}$, hepatitis $\mathrm{C}$, rheumatoid factor, anti-neutrophil cytoplasmic antibodies, antinuclear antibody, cryoglobulins, fasting glucose, serum protein electrophoresis, HIV, anticardiolipin antibodies, lupus anticoagulant, beta2-glycoprotein, factor $\mathrm{V}$ Leiden, and prothrombin gene were normal.

A chest radiograph revealed hyperexpanded lungs compatible with chronic obstructive pulmonary disease. Electrocardiogram and transthoracic echocardiogram, electromyography and nerve conduction studies were all normal. Angiography revealed loss of contrast flow to both ulnar arteries at the wrist level, poor filling of the superficial palmar arches, and no contrast flow to the left fifth and right second, third, and fourth digit arteries. Arteries supplying the thumbs were relatively spared. Subclavian, axillary, and brachial arteries were patent. In the right lower extremity there was no visible plantar arch and absent filling of distal arches. There were no corkscrew-like collateral vessels noted.

Upon admission, he was started on prednisone $1 \mathrm{mg} / \mathrm{kg} /$ day and low dose aspirin and continued on nifedipine. Patient was advised to stop smoking marijuana. His hand pain decreased and purplish discoloration to his hands began to regress. Prednisone was eventually tapered down to $5 \mathrm{mg}$ per day. Nevertheless, patient still required amputation of his left third and right fifth digits. Repeat arteriogram nine months later revealed slight progression and exam revealed purplish discoloration to his left thumb. Patient admitted to a recent lapse of marijuana usage. His urine drug screen tested positive for cannabis. 


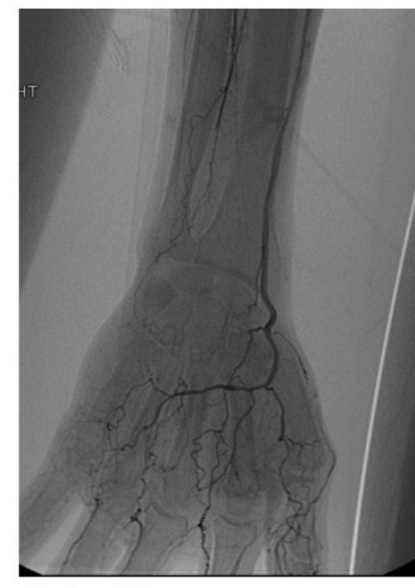

Picture 01

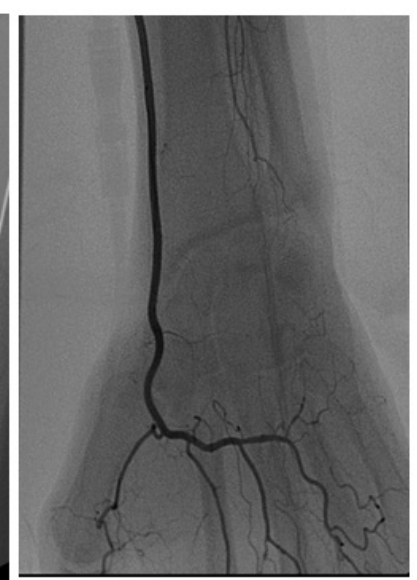

Picture 02

Legend for figures :

Arteriogram of right upper extremity (Picture 1) and left upper extremity (Picture 2) show occlusion of the ulnar artery at the level of the wrist. Note the poor filling of the superficial palmar arch on the ulnar side. The radial artery is mostly patent and blood flow to the thumbs is relatively preserved. Note the absence of collaterals or corkscrews.

\section{Case Two}

A Caucasian man in his 20's, presented with new onset of Reynaud's, for 8 months, followed by diffuse pain, prolonged joint stiffness, and intermittent tingling in median nerve distribution of both hands.

Patient admitted to smoking marijuana on daily basis since starting his job about 12 months, in a marijuana farm. During that time he avoided direct contact with pesticide and denied other illicit drugs. His initial exam revealed mildly dilated nail fold capillary without dropouts. Rest of exam was unremarkable. His labs revealed negative ANA scleroderma antibody, CCP, Rheumatoid factor, normal inflammatory markers, complete blood count, complete metabolic panel, and negative urinalysis. He later stopped both marijuana and working on farm. Despite this conservative treatment of avoidance, he continued to have arthralgia and Raynaud's. Importantly with stopping of marijuana he did not progress with digital ulceration.

\section{Discussion}

In our first case, several differential diagnoses were considered such as PolyarteritisNodosa (PAN), cryoglobulinemia, Buerger's disease. The lack of vasculitic rash, lack of peripheral neuropathy, undetectable viral load, and normal complements and negative serologic work up made these unlikely. Patient was diagnosed with Marijuana induced arteritis becausecessation of marijuana immediately improved symptoms. When he relapsed and began to use marijuana again his symptoms returned.

In the second case, Raynaud's occurred shortly after use of Marijuana. Although patient continued to have Raynaud's however did not progress to digital ischemia after stopping the offending agent.

Cannabis Arteritis was first described in 1960 in young Moroccan males who smoked kif, an oriental variety of cannabis extract, and was largely forgotten in the literature until the 1990s [6]. Since then, over 50 cases have been reported, mainly in European publications; it is generally believed that there is a vast underestimation of the actual prevalence of this condition. Cannabis Arteritis closely resembles Buerger's disease clinically and arteriographically and a correlation can

be made between the role of tobacco in Buerger's disease and cannabis in cannabis arteritis. A synergistic noxious effect of tobacco and cannabis has been postulated.

Cannabis Arteritis most commonly affects patients between the ages of 20 and 40, and typically presents with ischemic manifestation of the hands or feet. The lower extremities are more commonly affected. The review by Peyrot only identified 2 cases involving upper extremities and a review by Combemale likewise only identified 3 cases involving the upper extremities $[7,8]$. Commonly reported symptoms of cannabis arteritis include claudication, acral pain, recent Reynaud's phenomenon, and distal necrosis or gangrene of lower limbs. A history of cannabis use is always reported. There has been one case report of lower limb arteriopathy in a patient with significant amphetamine use and concomitant cannabis use as well, and the author has proposed that the arteriopathy was likely due to amphetamine via an inflammatory disturbance of the vasa vasorum, though the author also proposed a possible synergistic effect of amphetamine and cannabis [9]. Contaminants use of arsenic has been implicated in the Blackfoot disease, an endemic from of Buerger's disease in Taiwan related to chronic arsenic poisoning from well water [10].

On exam, there may be weak or absent distal pulses, Allen's maneuver can be positive with delay of filling of palmar arcades; acute phase reactants and autoantibodies are usually normal or negative. Laboratory tests for thrombophilic factors are often negative. In our case, lupus anticoagulant, anticardiolipin, and beta 2 glycoprotein were negative. Homocysteine was normal at 9.8 and Factor V Leiden and prothrombin G20210A were wildtype. In Disdier's review of ten patients, three were noted to have slightly elevated homocysteine levels, though the levels were not specified. Most patients did not have other cardiovascular risk factors [11]. In Peyrot's review of 55 patients, hypercholesterolemia was documented once and there were 4 cases of hypertriglyceridemia and one case of diabetes [7], though this was not observed in our patient.

Angiography often reveals distal segmental occlusions or skips lesions characterized by areas of diseased vessels interspersed with normal vessels. Both abrupt and smoothly tapered arterial occlusions can be found often with adjacent tortuous collaterals termed "corkscrews". Compensatory networks have been found to be less well developed with less observation of corkscrews in patients with cannabis arteritis than in patients with Buerger's Disease [12].

Histopathologic data is sparse and contradictory. Arterial biopsy is rarely performed as it may aggravate the condition. Sterne and Ducastaingt had reported that arterial biopsy in cannabis arteritis showed thrombosis, but no inflammation of the vascular wall. However, this histopathology analysis was made in late lesions and the author did not specify how many biopsies they found [13]. On the other hand, Disdier has reported thrombosis and endarteritis associated with mild inflammation involving the media, with fragmentation of the internal elastic lamina [11]

Pathogenesis remains unclear. Tetrahydrocannabinol, the main psychoactive component of cannabis, may induce vasoconstriction in several models.With cessation of cannabis, disease progression may be halted. In a series of 48 ischemic stroke patients aged less than 45 years, cannabis use appeared to be the most strongly associated factor with multifocal intracranial stenosis. The radiological characteristics of their angiopathy were the involvement of multiple intracranial arteries and the reversibility of vasoconstriction after cannabis withdrawal [14]. Furthermore symptoms of Raynaud's have been reported in cannabis smokers $[15,16]$. Currently there are no reports on cannabis induced connective tissue disease yet. Medical treatment comprises of vasodilator drugs (pentoxyfylline or iloprost) and anticoagulants in the acute phase followed by platelet aggregation inhibitors; aspirin 75-100 mg a day has been recommended for all patients [17]; severe disease has been treated with thrombolysis, arterial bypass and sometimes amputations. Surgical 
revascularization is generally not attempted because small peripheral arteries are most commonly involved; the only definitive treatment for cannabis arteritis is discontinuation of cannabis use.

The observation in these 2 cases, demonstrates that cannabis may represent a possible cofactor in the pathogenesis of Raynaud's, and arteritis.Due lack of follow up and unpublished reports, at this stage, we cannot comment on association between cannabis smokers and connective tissue disease.However, a number of authors have touted the importance of searching for cannabis use in all young patients with peripheral obstructive arteriopathy as cannabis weaning can lead to significant improvement in cannabis arteritis. This is even moretrue as cannabis usage is becoming legalized in more states and as the medical indications for marijuana continues to increase.

\section{References}

1. Abel EL. Cannabis in the Ancient World (chapter 1). Berlin: Springer Publications, 1980

2. United States Pharmacopeia, Appendix C. Antique Cannabis Book Website.

3. United Nations Office on Drugs and Crime. Single Convention on Narcotic Drugs.

4. Hasin DS, Saha TD, Kerridge BT. Prevalence of marijuana use disorders in the United States between 2001-2002 and 20122013. JAMA Psychiatry. 2015;72(12):1235-1245. doi:10.1001/ jamapsychiatry.2015.1858.

5. Center for Behavioral Health Statistics and Quality (CBHSQ).Substance Abuse and Mental Health Services Administration; 2015.

6. Nahas GG. Cannabis arteritis. N Engl J Med. 1971;284(2):113. DOI:10.1056/NEJM197101142840226.

7. Peyrot I, Garsaud AM, Saint-Cyr I, Quitman O, Sanchez B, et al. Cannabis arteritis: a new case report and a review of literature. Journal of the European Academy of Dermatology \& Venereology. 2007;21(3):388391.
8. Combemale P, Consort T, Denis-Thelis L, Estival JL, Dupin M, et al. Cannabis arteritis. Br.J.Dermatol. 2005;152(1):166-169.

9. Leithauser B, Langheinrich AC, Rau WS, Tillmanns H, Matthias FR. A 22-year-old woman with lower limb arteriopathy. Buerger's disease, or methamphetamine- or cannabis-induced arteritis? Heart \& Vessels. 2005;20(1):39-43. DOI: 10.1007/s00380-004-0769-5.

10. Chiou JM, Wang SL, Chen CJ, Deng CR, Lin W, et al. Arsenic ingestion and increased microvascular disease risk: observations from the south-western arseniasis-endemic area in Taiwan. Int J Epidemiol. 2005;34(4):936-943.

11. Disdier P, Granel B, Serratrice J, Constans J, Michon-Pasturel U, et al. Cannabis arteritis revisited--ten new case reports. Angiology. 2001;52(1):1-5. DOI:10.1177/000331970105200101.

12. Szuba A, Cooke JP. Thromboangiitis obliterans. An update on Buerger's disease.[see comment]. West.J.Med. 1998;168(4):255-260.

13. Sterne J, Ducastaing C. [Arteritis caused by Cannabis indica.]. Arch.Mal. Coeur.Vaiss. 1960;53:143-147.

14. Wolff V, Lauer V, Rouyer O, Sellal F, Meyer N, et al. Cannabis use, ischemic stroke, and multifocal intracranial vasoconstriction: a prospective study in 48 consecutive young patients. Stroke 2011;42:1778-1780. doi: 10.1161/STROKEAHA.110.610915.

15. Gröger A, Aslani A, Wolter T, Noah EM, Pallua N. Vasa. 2003;32(2):9597.

16. Tennstedt D, Saint-Remy A. Cannabis and skin diseases. Eur J Dermatol. 2011;21(1):5-11. doi:10.1684/ejd.2011.1212.

17. Noel B, Ruf I, Panizzon RG. Cannabis arteritis. J.Am.Acad.Dermatol. 2008;58(5 Suppl 1):S65-S67. doi: 10.1016/j.jaad.2007.04.024. 